\title{
AUTOMATIZACIÓN DEL PROCESO DE SECADO DE PRODUCTO HÚMEDO, USANDO UN MÉTODO DE CONTROL MULTIVARIABLE SIMPLIFICADO
}

\author{
Hernán Alonso López Ortiz ${ }^{1}$. Luis Edo García Jaimes ${ }^{2}$. \\ 1 Ingeniero en instrumentación y control, Politécnico Colombiano Jaime Isaza Cadavid, \\ hernan lopez54172@elpoli.edu.co. \\ ${ }^{2}$ MSc, docente Ingeniería en Instrumentación y Control, Politécnico Colombiano Jaime Isaza Cadavid, \\ luisgarcia@elpoli.edu.co
}

\begin{abstract}
RESUMEN
En este artículo se presenta el diseño e implementación de un sistema de control multivariable para un proceso productivo de secado de producto húmedo en un secador a chorro, utilizando controladores PI y desacopladores de tipo simplificado. El objetivo principal de esta implementación es reducir la cantidad de producto no conforme que se genera en una compañía del sector minero, por la manipulación manual de las variables de entrada y de salida, por la fuerte interacción entre ellas y por el efecto de las perturbaciones en el proceso que generan pérdidas tanto en tiempo como en el producto. Para el diseño se realizó la identificación del proceso, se estimaron los controladores PI fuera de línea y, finalmente se realizó el diseño de los desacopladores. Los resultados obtenidos a escala de simulación y en el proceso real demostraron la efectividad del sistema de control diseñado.
\end{abstract}

Palabras clave: Secado de producto húmedo, desacopladores, control multivariable, automatización.

Recibido: 30 de Septiembre de 2018. Aceptado: 28 de Diciembre de 2018

Received: September 30, 2018. Accepted: December 28, 2018

\section{AUTOMATION OF THE PROCESS OF DRYING WET PRODUCT, USING NO PARAMETRIC METHODS OF MULTIVARIABLE CONTROL}

\begin{abstract}
This paper presents the design and implementation of a multivariable system control for a productive process of drying of wet product on a Jet dryer, using PI controllers and simplified decoupling. The main goal of this implementation is to reduce the amount of non-compliant product that is generated at a company in the mining sector, for manual manipulation of input and output variables, by the strong interaction between them and the effect of the disturbances in the process that generated losses both in time and in the product. For the design, an identification of the process was carried out, then the PI controllers were estimated offline and finally the decoupling design was made. The results yielded from the simulation and the implementation in the process demonstrated the effectiveness of the designed control system.
\end{abstract}

Key words: Drying of wet product, decoupling, multivariable control, automation.

Cómo citar este artículo: H. Alonso, L. García, "Automatización del proceso de secado de producto húmedo, usando un método de control multivariable simplificado", Revista Politécnica, vol. 15, no.28 pp.20-31, 2019. DOI: https://doi.org/10.33571/rpolitec.v15n28a2 


\section{INTRODUCCIÓN}

Según [2], uno de los procesos más antiguos que existe en la industria es el de secado de materiales, cuya tecnología en las últimas décadas ha mejorado a causa de la necesidad de implementar procesos industriales más eficientes que reduzcan el consumo de energía y el tiempo empleado, y para alcanzar niveles más bajos de humedad en las partículas. Estos requerimientos se han alcanzado con la implementación de la automatización. En la literatura se referencian algunos trabajos importantes realizados alrededor de la automatización de secadores a chorro, en [3] se realizó el análisis de una planta para producir carbonato de calcio precipitado en la cual se modela y automatiza un prototipo de secador a chorro, en [4] se desarrolla una metodología de diseño simultáneo de proceso y control para un equipo de secado por atomización multiproducto para colorantes naturales, en [5] se utiliza una suspensión concentrada de partículas de carbonato de calcio para evaluar el efecto de la temperatura de secado sobre la microestructura de las microcápsulas de carbonato de calcio.

El trabajo se realizó en una empresa del sector minero que produce carbonato de calcio usando diferentes equipos y métodos, incluyendo el secado de material húmedo, proceso en el que se ve la necesidad de reducir los tiempos muertos generados por atascamientos y la cantidad de producto no conforme en un secador a chorro.

Con base en lo anterior, se desarrolló la automatización del proceso de secado de producto húmedo, usando un método de control multivariable simplificado, que atenúe la interacción entre las variables y el efecto de las perturbaciones generadas por otras condiciones en el mismo proceso y para evitar la manipulación manual de algunas de las variables que como consecuencia generaba atascamientos en el proceso.

El objetivo del trabajo consiste en automatizar el proceso de secado de carbonato de calcio, utilizando un método de control multivariable simplificado con el fin de reducir la interacción entre las variables. Para lograr lo anterior, se plantean como objetivos: definir las variables de entrada y salida del proceso y establecer la interacción que existe entre ellas, obtener las funciones de transferencia correspondientes a las variables de entrada y salida que presenten la mayor interacción, diseñar desacopladores que reduzcan la interacción entre dichas variables y sintonizar controladores PI para el control del sistema.

\section{MATERIALES Y MÉTODO}

Metodológicamente, el documento está organizado de la siguiente manera: se inicia con una breve descripción del proceso de secado en un secador a chorro, a continuación, se contextualiza el control multivariable y el uso de desacopladores para disminuir la interacción entre las variables del proceso, luego se presentan las funciones de transferencia del proceso obtenidas con las combinaciones de las variables controladas y manipuladas y se diseñan desacopladores simplificados y controladores PI para el controlar el proceso, finalmente, se realiza una comparación entre el desempeño del control multivariable implementado y el de la planta con la automatización inicial sin los desacopladores.

\subsection{MARCO CONCEPTUAL}

La empresa minera para la cual se desarrolló este proyecto tiene la necesidad de reducir la cantidad de producto no conforme producido en el secador a chorro, lo anterior por requerimiento en una auditoría realizada al departamento de producción, y definiendo la causa raíz del problema en la operación manual de diferentes variables en el proceso, a la falta de instrumentos de medida, la ausencia de automatización en algunos puntos del mismo, a la fuerte interacción entre las variables del proceso y a perturbaciones externas e internas que no son medidas.

En el estudio análisis de dicha auditoria se afirmó que todo lo anterior genera cambios en la dinámica del sistema y hace que el control implementado solo sea adecuado bajo condiciones específicas y que en el momento en el que se hacen presentes las perturbaciones, la operación es ineficiente provocando paros inesperados en el proceso y uso inadecuado de los recursos energéticos, llegando a contaminar el producto final. 


\subsubsection{Proceso de secado de producto húmedo}

En [6] el secado se define como una operación ubicua que se encuentra en casi todos los sectores industriales que van desde la agricultura hasta los productos farmacéuticos. La tecnología de secado implica el acoplamiento de los fenómenos del transporte y la ciencia de los materiales, ya que no se trata solo de la eliminación de líquido para producir un producto sólido, sino también del desarrollo de los criterios de calidad necesarios en el producto seco. A menudo, lo que es óptimo para el calor y las tasas de transferencia de masa no es apropiado para el secado de material húmedo. Además, para llegar a cumplir con las especificaciones del producto el secador debe contar con los instrumentos de medida apropiados y un control que las satisfaga.

El proceso de secado objeto de este proyecto es usado para secar un lodo o slurry que es alimentado al secador con un contenido entre 70 - 75\% de sólidos y $25-30 \%$ de agua, hasta obtener a la salida carbonato de calcio seco con un $0.01 \%$ de humedad a una tasa de producción entre 1800 y $2100 \mathrm{~kg} / \mathrm{h}$.

Para lograr lo anterior, el proceso cuenta con un tanque donde reside el slurry agitado para su homogenización, este material es transportado por una bomba de presión positiva a un flujo definido por la productividad requerida en $\mathrm{kg} / \mathrm{h}$, hasta una boquilla donde el slurry es mezclado con aire comprimido, obteniendo así una aspersión en tipo de nube en el cuerpo del secador; un quemador a gas con un ventilador a velocidad fija genera aire caliente para el secado del material, este aire puede alcanzar una temperatura entre los 480 y $700^{\circ} \mathrm{C}$ dependiendo de las condiciones de temperatura de salida del material y el flujo de alimentación, llegando al sector del secador donde se hace la aspersión del slurry. Después de pasar por el cuerpo del secador, el material es transportado a un colector de polvos que cuenta con un ventilador también a velocidad fija el cual determina el tiempo de residencia del material succionando el producto del cuerpo del secador para llevarlo a un filtro a una temperatura que esta entre 145 y $155^{\circ} \mathrm{C}$, entregándolo finalmente a un sistema de transporte neumático que lleva el material seco hasta los silos de producto terminado.
La instrumentación usada para el control del proceso consta de un sensor de temperatura en el área del quemador, con esta se hacen incrementos en el flujo de slurry hasta alcanzar la máxima temperatura permitida por el equipo, un sensor de temperatura a la entrada del colector de polvos, con este se hace el control del quemador para alcanzar la temperatura de salida deseada entre $145^{\circ} \mathrm{C}$ y $160^{\circ} \mathrm{C}$. Adicional a esto se tiene la medición de la humedad relativa a la salida de los gases, sensores de temperatura y presión para determinar la continuidad del flujo a la salida y las condiciones del proceso.

La Fig.1 muestra el diagrama de flujo del proceso de secado operativo.

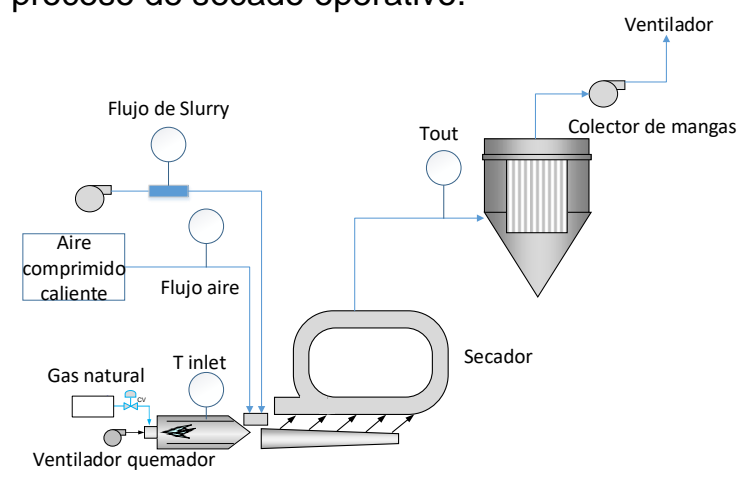

Fig. 1. Proceso de secado

\subsubsection{Proceso multivariable}

Los procesos multivariable son sistemas con varias entradas y varias salidas en los cuales una entrada afecta a diferentes salidas y al mismo tiempo, una salida es afectada por varias entradas, como muestra la Fig.2 para un sistema $2 X 2$ [7]. De acuerdo con [8], el control de estos procesos presenta dificultades debido a la interacción que existe entre los lazos de control.

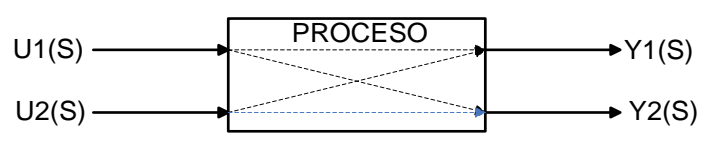

Fig. 2. Proceso multivariable $2 \times 2$

\subsubsection{Control multivariable}

Según [9], El principal objetivo al diseñar un sistema de control multivariable, para procesos industriales, es obtener una estructura que cumpla con las especificaciones de 
funcionamiento y que minimice la interacción entre sus variables. Por este motivo, una de las principales consideraciones que se hace al diseñar una estructura de control para procesos multivariables, es determinar la forma de emparejar las variables controladas y manipuladas, para formar los lazos de control, ya que con un adecuado emparejamiento se puede disminuir el efecto de las interacciones como se afirma en [10]. A continuación, se presenta la formulación teórica y los procedimientos aplicados para implementar un sistema de control multivariable a un proceso de dos entradas y dos salidas (TITO - Two Inputs, Two Outputs).

\subsubsection{Método de Bristol para estimar la interacción entre variables}

Una técnica utilizada para resolver el problema del emparejamiento entre variables manipuladas y controladas y que permite estimar el grado de las interacciones en régimen permanente, es el método de Bristol de las ganancias relativas propuesto en [9], [10] y [11]. Para aplicar este método es necesario obtener la matriz de ganancias relativas $(R G A)$, la cual se calcula a partir de la matriz de ganancias estáticas en lazo abierto K.

Las salidas $Y_{1}(S)$ y $Y_{2}(S)$ del sistema $2 \times 2$ de la Fig.2 se pueden escribir, en términos de las entradas $U_{1}(S)$ y $U_{2}(S)$ en forma matricial como se muestra en la ecuación 1 y 2 :

$$
\begin{aligned}
& Y(S)=G(S) U(S) \\
& {\left[\begin{array}{l}
Y_{1}(S) \\
Y_{2}(S)
\end{array}\right]=\left[\begin{array}{ll}
G_{11}(S) & G_{12}(S) \\
G_{21}(S) & G_{22}(S)
\end{array}\right]\left[\begin{array}{l}
U_{1}(S) \\
U_{2}(S)
\end{array}\right]}
\end{aligned}
$$

La matriz de ganancias estáticas del sistema se muestra en la ecuación 3:

$$
K=\lim _{S \rightarrow 0} G(S)=\left[\begin{array}{ll}
K_{11} & K_{12} \\
K_{21} & K_{22}
\end{array}\right]
$$

Una vez obtenida $K$, la matriz de ganancias relativas $(R G A)$, se calcula con la ecuación 4:

$$
\lambda=K \times\left(K^{-1}\right)^{T}=\left[\begin{array}{ll}
\lambda_{11} & \lambda_{12} \\
\lambda_{21} & \lambda_{22}
\end{array}\right]
$$

Donde el símbolo $\times$ significa que las matrices se multiplican elemento a elemento.
Según [9], la interpretación de los valores de los elementos de la matriz $R G A$ es:

- Si $\lambda_{i j}=1$. No hay interacción entre las variables.

- Si $\lambda_{i j}=0$. La variable manipulada $j$ no afecta a la variable controlada $i$.

- Si Si $0<\lambda_{i j}<1$, Hay interacción. Máxima interacción cuando $\lambda_{i j}=0.5$

- Si $\lambda_{i j}>1$, Cuanto más lejos de uno, más interacción.

- Si $\lambda_{i j}=\infty$. No es posible el control.

- Si $\lambda_{i j}<0$. Se tendrán respuestas dinámicas lentas y malas.

- Las variables se emparejan de forma que se elija el $\lambda_{i j}$ mayor.

\subsubsection{Desacopladores}

Los desacopladores son elementos de control utilizados con el objetivo de eliminar o reducir al máximo la interacción entre variables. En [12], se definen como funciones de transferencia o ganancias que se agregan al controlador y que descomponen el sistema multivariable en múltiples subsistemas de una sola variable, permitiendo establecer un sistema de control multivariable con controladores independientes para cada lazo de control, como se indica en la Fig.3. Por comodidad, en las figuras no se escribe el operador S de Laplace.

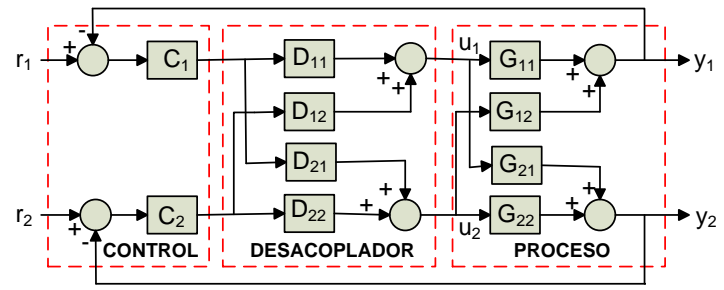

Fig. 3. Sistema multivariable con desacople

Existen básicamente dos métodos para el diseño: desacople dinámico cuando la red de desacople $D(S)$ se calcula utilizando las funciones de transferencia del proceso $G(S)$ y desacople estático cuando sólo utilizan sus ganancias en estado estacionario $\mathrm{G}(0)$. En este trabajo se analizan cuatro redes de desacople, dos del tipo dinámico y dos estáticas con el fin de comparar sus desempeños y seleccionar la más conveniente para el proceso.

\section{- Desacople simplificado.}


Se obtiene de la Fig.3. haciendo $D_{11}(S)=$ $D_{22}(S)=1$ y consiste en dos compensadores que sirven para minimizar el efecto de cada entrada del sistema con la salida que no le corresponde directamente como se indica en la Fig.4. Según [8], este tipo de desacople, permite el diseño de manera que el resultado sean elementos de desacople fáciles de implementar, teniendo en cuenta siempre, que sean realizables.

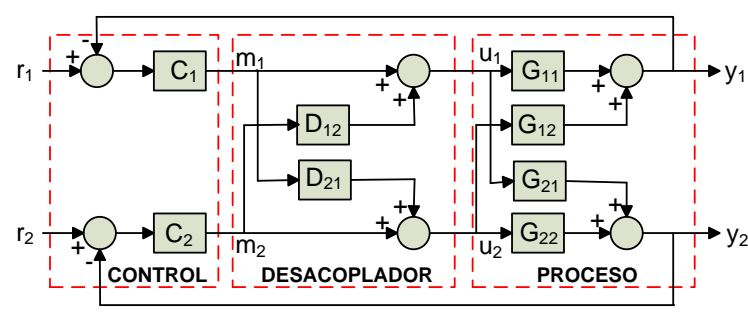

Fig. 4. Desacople simplificado dinámico

Utilizando el álgebra de diagramas de bloques se obtiene la ecuación 5:

$$
\begin{aligned}
& {\left[\begin{array}{l}
y_{1}(S) \\
y_{2}(S)
\end{array}\right]} \\
& =\left[\begin{array}{ll}
G_{11}(S)+D_{21}(S) G_{12}(S) & G_{12}(S)+D_{12}(S) G_{11}(S) \\
G_{21}(S)+D_{21}(S) G_{22}(S) & G_{22}(S)+D_{12}(S) G_{21}(S)
\end{array}\right]\left[\begin{array}{l}
m_{1}(S) \\
m_{2}(S)
\end{array}\right]
\end{aligned}
$$

Para que $y_{1}$ sea independiente de $m_{2}$ debe cumplirse que: $G_{12}(S)+D_{12}(S) G_{11}(S)=0$

Para que $y_{2}$ sea independiente de $m_{1}$ debe cumplirse que: $G_{21}(S)+D_{21}(S) G_{22}(S)=0$

Por lo tanto, la ecuación 6 expresa los valores resultantes para D12 y D21:

$$
D_{12}(S)=-\frac{G_{12}(S)}{G_{11}(S)} \quad D_{21}(S)=-\frac{G_{21}(S)}{G_{22}(S)}
$$

\section{- Desacople Simplificado de Estado Estacionario}

Se obtiene calculando la ganancia estática de cada una de las funciones de transferencia usadas en la determinación de los desacopladores simplificados, mostrados en la ecuación 7:

$$
K_{12}=-\frac{G_{12}(0)}{G_{11}(0)} \quad K_{21}=-\frac{G_{21}(0)}{G_{22}(0)}
$$

En la Fig.5. se muestra la implementación del desacople simplificado estacionario

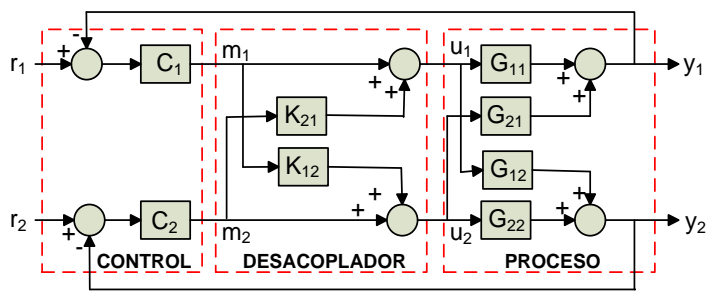

Fig. 5. Desacople simplificado estático

\section{- Desacople inverso}

Con este tipo de desacople, las funciones de transferencia de los elementos de la red de desacople son relativamente sencillas independientemente del tamaño del sistema como se afirma en [13]. El desacople inverso, obtiene la señal de control como una combinación de las señales de los controladores, donde una será la señal directa de uno de los controladores, mientras que la otra será la señal de la salida del otro controlador filtrada por los elementos de desacople como se indica en la Fig.6.

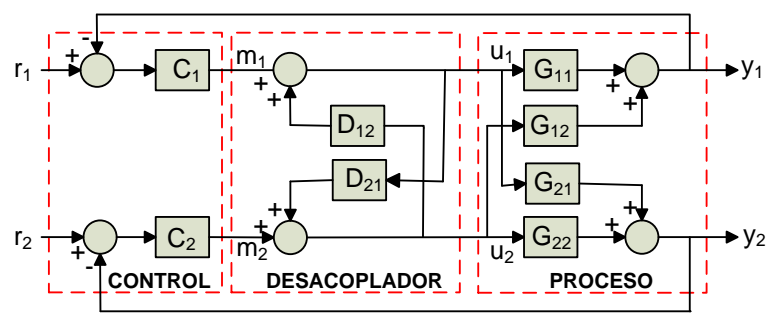

Fig. 6. Desacople inverso simplificado

Utilizando la fórmula de Mason se obtiene como resultado la ecuación 8 :

$\left[\begin{array}{l}y_{1}(S) \\ y_{2}(S)\end{array}\right]$

$=\frac{\left[\begin{array}{ll}G_{11}(S)+D_{21}(S) G_{12}(S) & G_{12}(S)+D_{12}(S) G_{11}(S) \\ G_{21}(S)+D_{21}(S) G_{22}(S) & G_{22}(S)+D_{12}(S) G_{21}(S)\end{array}\right]\left[\begin{array}{c}m_{1}(S) \\ m_{2}(S)\end{array}\right]}{1-D_{12}(S) D_{22}(S)}$

Para que $y_{1}$ sea independiente de $m_{2}$ debe cumplirse que: $G_{21}(S)+D_{12}(S) G_{11}(S)=0$

Para que $y_{2}$ sea independiente de $m_{1}$ debe cumplirse que: $G_{12}(S)+D_{21}(S) G_{22}(S)=0$

Por lo tanto, la ecuación 9 expresa los valores resultantes para D12 y D21: 


$$
D_{12}=-\frac{G_{12}(S)}{G_{11}(S)} \quad D_{21}=-\frac{G_{21}(S)}{G_{22}(S)}
$$

\section{- Desacople inverso estático}

Para estimar este tipo de desacople basta calcular la ganancia estática de cada una de las funciones de transferencia de la red de desacoplo inverso, como se muestra en la ecuación 10 e indica en la Fig.7.

$$
K_{12}=-\frac{G_{12}(0)}{G_{11}(0)} \quad K_{21}=-\frac{G_{21}(0)}{G_{22}(0)}
$$

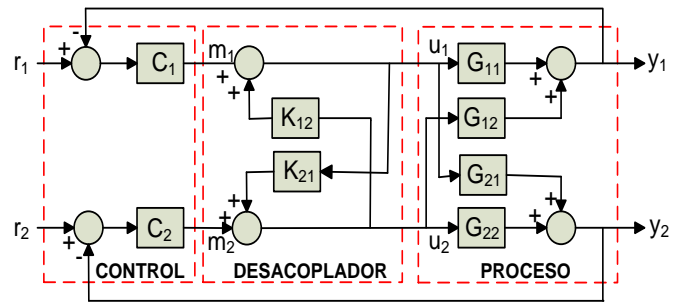

Fig. 7. Desacople inverso estático

\subsubsection{Diseño del controlador}

Para sintonizar los controladores se utilizó el método de asignación de polos. Como los lazos se encuentran desacoplados, es posible sintonizar cada controlador de manera independiente. En [14] y [15] se afirma que, si las funciones de transferencias del sistema son de tipo cero, es conveniente utilizar controladores de tipo $\mathrm{PI}$, con el fin de reducir a cero o a un valor mínimo aceptable, el error de estado estable.

La función de transferencia de lazo cerrado se muestra en la ecuación 11 :

$G_{w}(S)=\frac{G_{c}(S) G_{P}(S)}{1+G_{c}(S) G_{P}(S)}$

La ecuación del controlador PI se puede escribir como se indica en la ecuación 12:

$G_{c}(S)=K_{c}+\frac{K_{i}}{S}$

Si se asignan dos polos en $S=P=-\xi \omega_{n} \pm$ $j w_{n} \sqrt{1-\xi^{2}}$

La ecuación característica del sistema en lazo cerrado cumple con lo mostrado en la ecuación 13:

$$
1+\left(K_{c}+\frac{K_{i}}{P}\right) G_{P}(P)=0
$$

Resolviendo la ecuación 13 para $K_{c}$ y $K_{i}$ da como resultado las ecuaciones 14 y 15 :

$\begin{aligned} K_{c} & =\frac{A\left(\omega_{n}\right) \sqrt{1-\xi^{2}}+\xi B\left(\omega_{n}\right)}{\sqrt{1-\xi^{2}}\left(A^{2}\left(\omega_{n}\right)+B^{2}\left(\omega_{n}\right)\right)} \\ K_{i} & =\frac{\omega_{n} B\left(\omega_{n}\right)}{\sqrt{1-\xi^{2}}\left(A^{2}\left(\omega_{n}\right)+B^{2}\left(\omega_{n}\right)\right)}\end{aligned}$

En donde:

$$
A\left(\omega_{n}\right)=\operatorname{Re}\left[G_{P}(P)\right] \quad B\left(\omega_{n}\right)=\operatorname{Im}\left[G_{P}(P)\right]
$$

\section{RESULTADOS}

\subsection{MODELO MATEMÁTICO DEL SECADOR}

Para analizar la interacción entre las variables de entrada y de salida del proceso, se realizó un análisis estadístico de correlación con los datos obtenidos en el trabajo normal del sistema. Los resultados se muestran en la Tabla 1.

Tabla 1. Correlación entre variables de entrada y variables de salida del secador

\begin{tabular}{lcc}
\hline & \multicolumn{2}{c}{ ENTRADAS } \\
\cline { 2 - 3 } \multicolumn{1}{c}{ SALIDAS } & $\begin{array}{c}\text { Flujo de } \\
\text { slurry } \\
(\mathrm{l} / \mathrm{m})\end{array}$ & $\begin{array}{c}\text { Flujo } \\
\text { de } \\
\text { gas } \\
(\%)\end{array}$ \\
\hline $\begin{array}{l}\text { Temp. entrada al } \\
\text { Quemador }\left({ }^{\circ} \mathrm{C}\right)\end{array}$ & 0.978 & 0.816 \\
$\begin{array}{l}\text { Temp. salida del proceso } \\
\left({ }^{\circ} \mathrm{C}\right)\end{array}$ & 0.474 & 0.679 \\
$\begin{array}{l}\text { Temp. entrada al filtro }\left({ }^{\circ} \mathrm{C}\right) \\
\text { Humedad salida gases } \\
\text { filtro }(\%)\end{array}$ & 0.463 & 0.693 \\
$\begin{array}{l}\text { Temp. salida del } \\
\text { Quemador }\left({ }^{\circ} \mathrm{C}\right)\end{array}$ & 0.152 & -0.026 \\
\hline
\end{tabular}

De los datos obtenidos en el análisis de correlación, se toman como entradas: el flujo de slurry (u1) y el flujo de gas, $(u 2)$ y como salidas la temperatura a la entrada del quemador $\left(T_{i n}\right)$ y la temperatura de salida del secador $\left(T_{\text {out }}\right)$. Al establecer la interacción entre estas variables se 
obtuvieron, mediante identificación a partir de la curva de reacción, las funciones de transferencia resultantes de la combinación de las dos entradas con las dos salidas, como se muestran en las ecuaciones 16 a 19:

$$
\begin{aligned}
& G_{11}(S)=\frac{T_{\text {out }}(S)}{U_{1}(S)}=\frac{0.0432(S+0.0122)}{(S+0.01464)(S+0.0043)} \\
& G_{12}(S)=\frac{T_{\text {out }}(S)}{U_{2}(S)}=\frac{0.0464(S+0.00116)}{(S+0.00808)(S+0.00678)} \\
& G_{21}(S)=\frac{T_{\text {in }}(S)}{U_{1}(S)}=\frac{1.237(S+0.0144)}{(S+0.2275)(S+0.00275)} \\
& G_{22}(S)=\frac{T_{\text {in }}(S)}{U_{2}(S)}=\frac{0.164(S+0.0122)}{(S+0.0288)(S+0.00515)}
\end{aligned}
$$

Para evaluar las interacciones entre variables manipuladas y variables controladas en régimen permanente se utilizó la matriz de ganancias estáticas del sistema dada por la ecuación 3.

Para el horno de secado queda en la forma expresada en las ecuaciones 20 y 21 :

$$
\begin{gathered}
{\left[\begin{array}{c}
T_{\text {out }} \\
T_{\text {in }}
\end{array}\right]=\left[\begin{array}{ll}
G_{11} & G_{12} \\
G_{21} & G_{22}
\end{array}\right]\left[\begin{array}{l}
u_{1} \\
u_{2}
\end{array}\right]} \\
K=\left[\begin{array}{cc}
8.372 & 0.982 \\
28.472 & 13.489
\end{array}\right]
\end{gathered}
$$

Una vez obtenida $K$, la matriz de ganancias relativas $(R G A) \lambda$, puede calcularse mediante la ecuación 4 , dando como resultado lo expresado en la ecuación 22:

$\lambda=\left[\begin{array}{cc}1.329 & -0.329 \\ -0.329 & 1.329\end{array}\right]$

De la RGA se observa que $\lambda_{12}<0$ y $\lambda_{21}<0$, y que $\lambda_{11}>1$ y $\lambda_{22}>1$ con dos últimos valores las interacciones son significativas por lo tanto, las mejores parejas de variable controlada variable manipulada son: $T_{\text {out }}-u_{1}$ y $T_{\text {in }}-u_{2}$.

En estas condiciones, el sistema completo con los controladores y la red de desacople queda como se indica en la Fig.8.

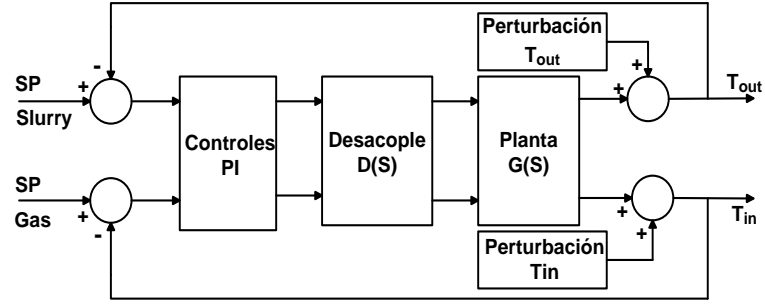

Fig. 8. Implementación de los controladores y la red de desacople

\subsection{DISEÑO DE LAS REDES DE DESACOPLE}

A continuación, en las ecuaciones 23 a 28 se presentan las redes de desacople obtenidas.

Red de desacople simplificada dinámica:

$$
\begin{aligned}
& D_{12}=-\frac{G_{12}}{G_{11}} \\
& =-\frac{1.074(S+0.00116)(S+0.01464)(S+0.0043)}{(S+0.0122)(S+0.00808)(S+0.00678)} \\
& D_{21}=-\frac{G_{21}}{G_{22}} \\
& =-\frac{7.542(S+0.0144)(S+0.0288)(S+0.00515)}{(S+0.2275)(S+0.00275)(S+0.0122)}
\end{aligned}
$$

Red de desacople simplificada estática:

$K_{12}=D_{12}(0)=-0.117 \quad K_{21}=D_{21}(0)=-2.11$

\section{Red simplificada inversa:}

$D_{12}=-\frac{G_{12}}{G_{11}}$

$=-\frac{1.074(S+0.00116)(S+0.01464)(S+0.0043)}{(S+0.0122)(S+0.00808)(S+0.00678)}$

$D_{21}=-\frac{G_{21}}{G_{22}}$

$=-\frac{7.542(S+0.0144)(S+0.0288)(S+0.00515)}{(S+0.2275)(S+0.00275)(S+0.0122)}$

\section{Red simplificada inversa estática:}

$K_{12}=D_{12}(0)=-0.117 \quad K_{21}=D_{21}(0)=-2.11$

\subsection{SINTONÍA DE LOS CONTROLADORES PI}

La función de transferencia $G_{11}(S)$ que relaciona el flujo de slurry $(u 1)$ y la temperatura de salida del secador $\left(T_{\text {in }}\right)$, tiene tiempo de establecimiento $t_{s 11}=1200 \mathrm{~s}$. Para asignar los polos deseados de lazo cerrado, se asume que $t_{s}=1000 \mathrm{~s}$ y coeficiente de amortiguamiento $\xi=0.8$ con el fin de garantizar una respuesta un poco más rápida que la correspondiente al 
proceso en lazo abierto. Con estas condiciones, el sistema tiene polos dominantes de lazo cerrado en $S=-0.00333 \pm J 0.0025$. El controlador PI para este proceso es el expresado en la ecuación 29:

$G_{C 1}(S)=0.1269+\frac{0.000493}{S}$

Por otro lado, la función de transferencia $G_{22}(S)$ que relaciona el flujo de gas $(u 2)$ y la temperatura de entrada del quemador $\left(T_{i n}\right)$, tiene tiempo de establecimiento $t_{s 22}=916 \mathrm{~s}$. Para asignar los polos deseados de lazo cerrado se asume, $t_{s}=800 \mathrm{~s}$ y coeficiente de amortiguamiento $\xi=0.8$, logrando así mayor velocidad de respuesta en el proceso. Con esas condiciones, el sistema tiene polos dominantes de lazo cerrado en $S=-0.005 \pm J 0.00375$. El controlador PI para este proceso es el expresado en la ecuación 30:

$G_{C 2}(S)=0.1088+\frac{0.000661}{S}$

En las Fig. 9 a 12 se muestran las respuestas de las variables controladas del horno ante cambios en el setpoint con los diferentes tipos de desacopladores diseñados. Los cambios se realizaron bajo las mismas condiciones de trabajo.

En la Fig. 9 se observa el sistema con los controladores $\mathrm{PI}$ sintonizados, pero sin desacopladores. Se observa que un cambio en la referencia del flujo de slurry afecta inmediatamente la temperatura mientras que el cambio en la referencia de esta variable prácticamente no tiene incidencia sobre el flujo de slurry.

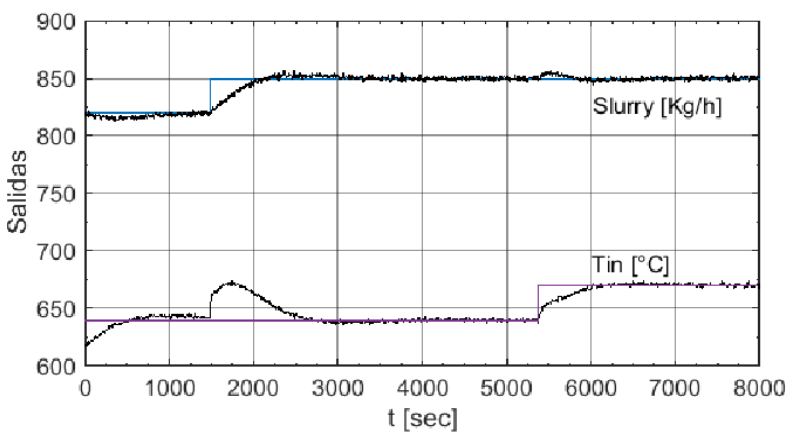

Fig. 9. sistema de control sin desacople

La Fig.10 corresponde al sistema con los controladores PI sintonizados y con desacople dinámico simplificado. Se observa que un cambio en la referencia del flujo de slurry afecta la temperatura, pero, a diferencia del caso anterior, en este la temperatura del quemador desciende y luego se estabiliza en el valor de referencia. Igualmente, un cambio en la referencia de esta variable no genera cambios significativos en el flujo de slurry.

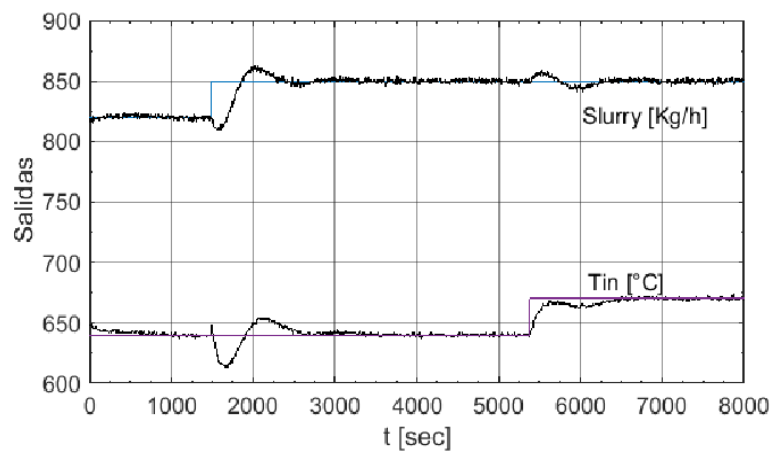

Fig. 10. Sistema con desacople simple dinámico

La Fg.11 corresponde al sistema con los controladores PI y desacople estático simplificado, al igual que en el caso anterior, un cambio en la referencia del flujo de slurry afecta la temperatura la cual experimenta un cambio transitorio significativo y luego se estabiliza en el valor de referencia. Así mismo, un cambio en la referencia de esta variable no genera cambios en el flujo de slurry.

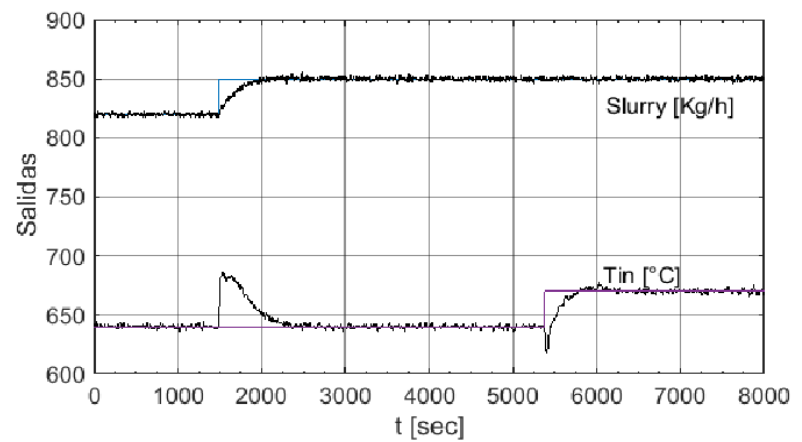

Fig. 11. Sistema con desacople simple estático

La Fig.12 muestra la respuesta del proceso con los controladores $\mathrm{PI}$ y con desacople inverso dinámico. Se observa que un cambio en la referencia del flujo de slurry afecta la temperatura que luego se estabiliza en el valor de referencia, además, en este caso, un cambio en la temperatura genera pequeños cambios transitorios en el flujo de slurry. 


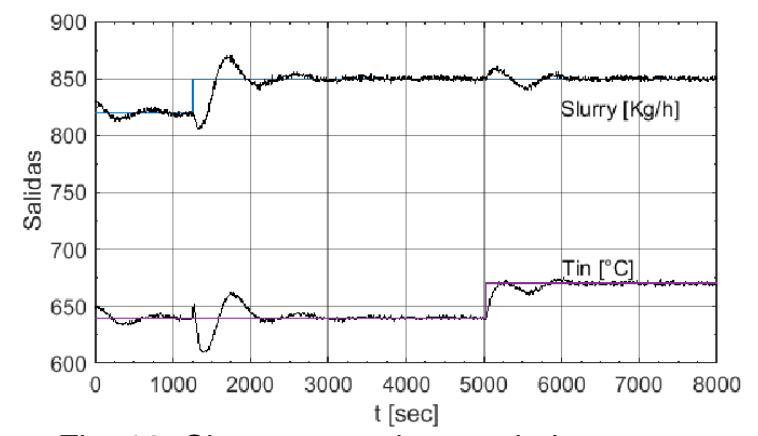

Fig. 12. Sistema con desacople inverso dinámico

Finalmente, cuando se utiliza desacople inverso estático, los cambios en el flujo de slurry no afectan en forma significativa la temperatura del quemador, sin embargo, en este caso, al aumentar la temperatura del quemador se presenta incremento momentáneo significativo en el flujo de slurry, pero con el tiempo retorna al valor de referencia, como se muestra más adelante en la Fig. 13.

\section{ANÁLISIS DE RESULTADOS}

Para establecer una comparación cuantitativa del desempeño de los diferentes tipos de desacopladores diseñados, se utilizaron métricas basadas en el error: integral del error absoluto (IAE), integral del cuadrado del error (ICE) y el error medio (EM), éste último medido en la zona de estabilización definitiva del proceso. También se utilizaron criterios de respuesta temporal para identificar el sistema con el menor tiempo de establecimiento, menor sobreimpulso y menor error de estado estable.

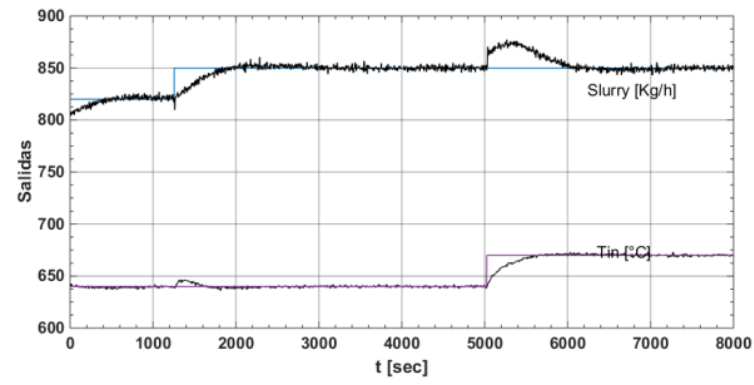

Fig. 13. Sistema con desacople inverso estático

En la Tabla 2 se presentan los valores estimados para cada uno de los criterios de comparación establecidos al introducir un cambio en la referencia del flujo de slurry. EI IAE y el ICE se miden desde antes de aplicar el cambio de referencia y hasta que el sistema se estabilice. EI EM se mide con el promedio de los últimos datos una vez estabilizado el proceso, es decir, hace referencia al error medio de estado estable. Atendiendo a este último criterio, el sistema de mejor desempeño es el DID, pero si se analizan los criterios de la integral del error, el sistema con mejores resultados es el DSE que sería el recomendado.

Tabla 2. Criterios de error para el flujo se slurry

\begin{tabular}{lccc}
\hline \multicolumn{1}{c}{ TIPO } & IAE & ICE & EM $[\mathrm{Kg} / \mathrm{h}]$ \\
\hline Sin Des & $1.18 * 10^{4}$ & $1.63 * 10^{5}$ & 0.947 \\
DSD & $1.29 * 10^{4}$ & $3.51 * 10^{5}$ & 0.917 \\
DSE & $8.36 * 10^{3}$ & $1.02 * 10^{5}$ & 0.937 \\
DID & $1.78 * 10^{4}$ & $3.92 * 10^{5}$ & 0.871 \\
DIE & $1.18 * 10^{4}$ & $1.59 * 10^{5}$ & 1.222 \\
\hline
\end{tabular}

Sin Des: Sistema sin desacople.

DSD: Desacople simplificado dinámico.

DSE: Desacople simplificado estático.

DID: Sistema con desacople inverso dinámico.

DIE: Sistema con desacople inverso estático.

En la Tabla 3 se presentan los valores estimados para cada uno de los criterios de comparación establecidos al introducir un cambio en la referencia de la temperatura del quemador. En este caso, el mejor desempeño lo presenta el DID.

Tabla 3. Criterios de error para la temperatura en el quemador

\begin{tabular}{lccc}
\hline \multicolumn{1}{c}{ TIPO } & IAE & ICE & EM $\left[{ }^{\circ} \mathrm{C}\right]$ \\
\hline Sin Des & $8.18 * 10^{3}$ & $4.63 * 10^{4}$ & 1.071 \\
DSD & $7.63 * 10^{3}$ & $7.17 * 10^{4}$ & 0.898 \\
DSE & $9.21 * 10^{3}$ & $2.06 * 10^{5}$ & 0.852 \\
DID & $6.14 * 10^{3}$ & $5.65 * 10^{4}$ & 0.885 \\
DIE & $7.18 * 10^{3}$ & $8.93 * 10^{4}$ & 0.637 \\
\hline
\end{tabular}

En la Tabla 4 se presentan algunas características de la respuesta temporal del proceso de flujo de slurry con los diferentes esquemas de desacople utilizados. Se aprecia que para los casos del DSD y del DID, el sobreimpulso es muy alto con respecto al propuesto en el diseño. Los esquemas de control restantes están dentro de los parámetros de diseño. 
Tabla 4. Parámetros de respuesta temporal para el flujo de slurry

\begin{tabular}{lccc}
\hline TIPO & $t_{s}[\mathrm{sec}]$ & $M p \%$ & $e_{S s} \%$ \\
\hline Sin Des & 1000 & 2 & 0 \\
DSD & 1200 & 33 & 0 \\
DSE & 1000 & 1 & 0 \\
DID & 1200 & 40 & 0 \\
DIE & 1200 & 1 & 0 \\
\hline
\end{tabular}

En la Tabla 5 se muestran resultados de las características de la respuesta temporal del proceso de temperatura en el quemador con los diferentes esquemas de desacople utilizados. Se observa que, para esta variable, los esquemas de control propuestos están dentro de los parámetros de diseño.

Tabla 5. Parámetros de respuesta temporal para la temperatura del quemador

\begin{tabular}{lccc}
\hline TIPO & $t_{s}[\mathrm{sec}]$ & $M p \%$ & $e_{s s} \%$ \\
\hline Sin Des & 800 & 0 & 0 \\
DSD & 1000 & 0 & 0 \\
DSE & 800 & 2 & 0 \\
DID & 1000 & 2 & 0 \\
DIE & 1000 & 2 & 0 \\
\hline
\end{tabular}

\section{CONCLUSIONES}

El diseño utilizado garantiza la estabilidad del sistema en lazo cerrado y los controladores del tipo PI aseguran error de estado estable igual a cero. El proyecto deja en claro que el control multivariable por desacoplo es una técnica que da muy buenos resultados cuando la interacción entre variables es fuerte y que mejora el desempeño del proceso.

Al finalizar el proyecto, se verifica que se ha cumplido el objetivo de comprobar las ventajas y limitantes de las estrategias de control multivariable aplicando redes de desacoplo en el control del secador a chorro utilizado en la producción de carbonato de calcio.

Se presentan resultados de simulación, utilizando un modelo lineal del sistema, obtenido mediante identificación no paramétrica con datos experimentales del proceso. Los ensayos realizados muestran que el sistema tiene un buen desempeño y que los controladores y el sistema de desacople responden adecuadamente ante cambios ocasionados en diferentes puntos de operación.
Los desacopladores se calcularon utilizando un modelo matemático lineal de la planta $2 \times 2$ obtenido en forma experimental y se implementaron controladores PI para regular las variables de salida utilizando la técnica de asignación de polos con lo cual se logró eliminar en forma adecuada la fuerte interacción entre las variables del proceso.

En las Tablas 1 y 2 se presentan los valores IAE e ICE como índices de desempeño. Los controles basados en la estructura DSE y DID son los que muestran el mejor comportamiento, las otras estructuras de desacoplo utilizadas presentan valores un poco más altos, pero en general, su desempeño es aceptable.

Finalmente, y a diferencia de otros trabajos sobre control de secadores a chorro en los cuales se controlan en forma independiente las variables, en éste, el control multivariable diseñado permite disminuir en forma significativa la interacción entre las variables y atenuar las perturbaciones.

\section{REFERENCIAS}

[1] Vázquez, F. Y Morilla. F. (2002). Tuning decentralized PID controllers for MIMO systems with decoupling. In 15th IFAC World Congress, pp. 2172-2178.

[2] Peep L, Rebassa J, Kartushinsky A, Tisler S, Tähemaa T, and Polonsky A. (2016) Assemblage of turbulent jet flows through static particulate media. Proceedings of the Estonian Academy of Sciences. 65(3), 284-296.

[3] Collado E, Domínguez P, Pizarro A (2000). Estudio técnico y económico de una planta para producir carbonato de calcio precipitado utilizado en la industria de los polímeros. Universidad Nacional de Ingeniería. Programa académico de ingen1eria de petróleo y petroquímica.

[4] Vera, M. (2012) Metodología de Diseño Simultáneo de Proceso y Control aplicada a un secado por atomización multiproducto para sustancias químicas naturales. Universidad Nacional de Colombia Facultad de Minas, Escuela de Procesos y Energía Medellín, Colombia 
[5] Diosa, J. Casanova, H (2015) Cambios microestructurales de agregados de carbonato de calcio generados a partir de secado por aspersión. 1 Grupo de Coloides, Facultad de Ciencias Exactas y Naturales, Universidad de Antioquia, A.A.1226, Medellín.

[6] Tadeusz Kudra, Arun S. Mujumdar. Advanced Drying Technologies, 2a. ed., Taylor \& Francis Group. Boca Raiton, London, New York, 2009.

[7] Morilla, F. Vázquez, f. Garrido, J. (2015). Control centralizado PID 2x2 por desacoplo. Departamento de Informática y Análisis Numérico, Universidad de Córdoba, Campus de Rabanales, 14071 Córdoba.

[8] Torreblanca, A. (2017) Desarrollo de un procedimiento teórico-práctico para el conocimiento y experimentación de sistemas multivariables particularmente en el caso de 4 tanques para fines didácticos. Tesis de grado. Universidad nacional San Agustín. Facultad de Ingeniería.

[9] Ospina, M. (2009). Diseño de un sistema de control multivariable para un circuito de molienda en húmedo. Tesis de Maestría. Universidad Nacional de Colombia. Facultad de Minas. Escuela de Ingeniería de Materiales Medellín.

[10] Garrido. (2012) Diseño de sistemas de control multivariable por desacoplo con controladores PID. Tesis Doctoral. Universidad Nacional de Educación a distancia. Departamento de Informática y Automática. Madrid.

[11] Bristol, E. H. (1966). On a New Measure of Interaction for Multivariable Process Control. IEEE Transactions on Automatic Control, 11, 133-134.

[12] Ramírez, R (2012). Análisis dinámico y control multivariable del sistema de tanques interconectados Feedback 33-230. Tesis de Maestría. Universidad de Costa Rica

Facultad de Ingeniería. Escuela de Ingeniería Eléctrica

[13] Orellana, R. Coronel, M. Rojas, R. (2017). Effect of decoupling techniques in multivariable systems. Universidad de Los Andes (ULA),
Venezuela. ISSN: 0122-3461 (impreso). 21459371 (on line)

[14] Vázquez, F., Morilla, F. (2002). Tuning decentralized PID controllers for MIMO systems with decouplers. Proceedings of the 15th IFAC World Congress. Barcelona, Spain.

[15] Vázquez, F., Morilla, F., Dormido, S. (1999). An iterative method for tuning decentralized PID controllers. Proceedings of the 14th IFAC World Congress. Beijing, China.

[16] Montero, L (2006). Sintonía de Reguladores PID en Sistemas Multivariables. Universidad Central "Marta Abreu" de Las Villas. Facultad de Ingeniería Eléctrica. Santa Clara. Cuba 